Part II

\title{
Supernovae: Observations
}




\section{Radio Supernovae}

Richard A. Sramek ${ }^{1}$, Kurt W. Weiler ${ }^{2}$, and Nino Panagia ${ }^{3}$

${ }^{1}$ NRAO, Socorro, NM, USA;

dsramek@aoc.nrao.edu, mrupen@aoc.nrao.edu

2 NRL, Washington, DC, USA;

Kurt. Weiler@nrl. navy. mil

${ }^{3}$ STScI, Baltimore, MD, USA and Astrophysics Division, Space Science Department of ESA;

panagia@stsci.edu

Summary. Detailed, multi-frequency radio observations of supernovae have shown that the radio emission can be understood as a blastwave interacting with a structured circumstellar medium (CSM) and modeling of the radio light curves allows estimation of the physical conditions in this medium. CSM structures, properties of the presupernova system, and the evolution of the system in the last stages before explosion can then be derived.

\section{Introduction}

Since 1970 , the study of radio emission from extragalactic supernovae has resulted in the detection of thirty-four objects; 24 Type II of various subclasses, no Type Ia supernovae, 9 Type Ib and Ic, plus one object, SN1982aa for which there is no optical type established [10]. These detections were among the 155 supernovae studied by mid 2003. Almost all of these detections were made in the past 20 years using the Very Large Array $(V L A)^{1}$ radio telescope.

Out of this extensive study of the radio emission from supernovae, several general observations can be made:

* type Ia SNe are not radio emitters to the detection limit of the VLA;

* type $\mathrm{Ib} / \mathrm{c}$ SNe are radio luminous with steep spectral indices (generally $\alpha<-1 ; S \propto \nu^{+\alpha}$ ) and a fast turn-on/turn-off, usually peaking at $6 \mathrm{~cm}$ near or before optical maximum;

* type II SNe show a range of radio luminosities with flatter spectral indices (generally $\alpha>-1$ ) and a relatively slow turn-on/turn-off, usually peaking at $6 \mathrm{~cm}$ significantly after optical maximum;

* type $\mathrm{Ib} / \mathrm{c}$ may be fairly homogeneous in some of their radio properties while type II, as in the optical, are quite diverse.

${ }^{1}$ The VLA telescope of the National Radio Astronomy Observatory is operated by Associated Universities, Inc. under a cooperative agreement with the National Science Foundation. 


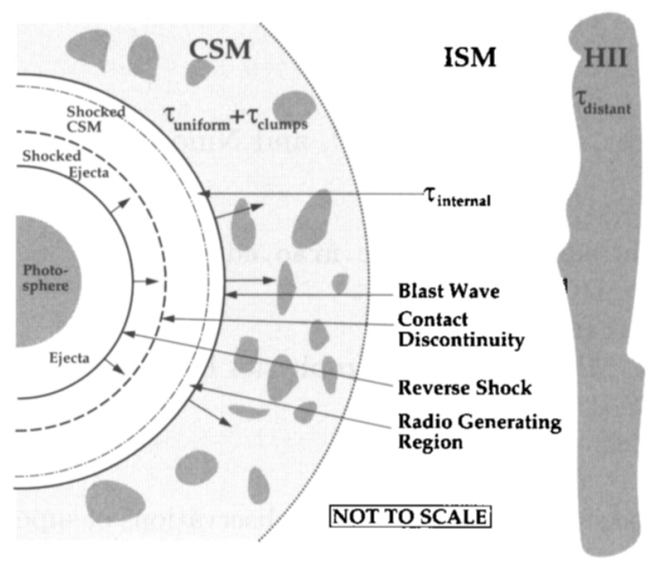

Fig. 1. Cartoon, not to scale, of the SN and its shocks, along with the stellar wind established circumstellar medium (CSM), the interstellar medium (ISM), and more distant ionized hydrogen (HII) absorbing gas. The radio emission is thought to arise near the blastwave front. The expected locations of the several absorbing terms in (1)-(10) are illustrated.

From the radio data it is possible to classify the properties of supernovae and to develop and test models for the radio emission which match the light curves. Among the results that come out of this work are estimates of the density and structure of the circumstellar material around supernovae, the density evolution of the presupernova stellar wind, and insight into the last stages of stellar evolution before the explosion.

\section{Parameterized Light Curves for Radio Supernovae}

The common characteristics that need to be incorporated in a radio supernova (RSN) model are:

* Nonthermal synchrotron emission with high brightness temperature;

* A decrease in absorption with time, resulting in a smooth, rapid turn-on first at shorter wavelengths and later at longer wavelengths;

* A power-law decline of the flux density with time at each wavelength after maximum flux density (optical depth $\sim 1$ ) is reached at that wavelength;

* A final, asymptotic approach of the spectral index $\alpha\left(S \propto \nu^{+\alpha}\right)$ to an optically thin, nonthermal, constant negative value $[12,13]$.

The characteristic RSN radio light curves (see, e.g., Fig. 2) arise from the competing effects of slowly declining non-thermal radio emission and more rapidly declining thermal or non-thermal absorption yielding a rapid turnon and slower turn-off of the radio emission at any single frequency. Since 
absorption processes are greater at lower frequencies, transition from optically thick to optically thin (turn-on) occurs first at higher frequencies and later at lower frequencies. After the radiation is completely optically thin and showing the ongoing decline of the underlying emission process (turn-off), the non-thermal spectrum causes lower frequencies to have higher flux density. These two effects cause the displacement in time and flux density of the light curves at different frequencies. Chevalier $[1,2]$ has proposed that the relativistic electrons and enhanced magnetic field necessary for synchrotron emission arise from the $\mathrm{SN}$ blastwave interacting with a relatively high density circumstellar medium which has been ionized and heated by the initial UV/Xray flash.

Weiler et al. [12, 13] and Montes et al. [5] adopted a parameterized model which has been updated in Weiler et al. $[17,18]$ to the following form:

$$
\begin{aligned}
F_{\nu}(\mathrm{mJy})= & K_{1}\left(\frac{\nu}{5 \mathrm{GHz}}\right)^{\alpha}\left(\frac{t-t_{0}}{1 \text { day }}\right)^{\beta} e^{-\tau_{\text {external }}}\left(\frac{1-e^{-\tau_{\mathrm{CSM}} \mathrm{clumps}_{\mathrm{c}}}}{\tau_{\mathrm{CSM}_{\mathrm{clumps}}}}\right) \times \\
& \left(\frac{1-e^{-\tau_{\text {internal }}}}{\tau_{\text {internal }}}\right)
\end{aligned}
$$

with

$$
\tau_{\text {external }}=\tau_{\mathrm{CSM}_{\text {homog }}}+\tau_{\text {distant }}
$$

where

$$
\begin{gathered}
\tau_{\mathrm{CSM}_{\mathrm{hom} O \mathrm{~g}}}=K_{2}\left(\frac{\nu}{5 \mathrm{GHz}}\right)^{-2.1}\left(\frac{t-t_{0}}{1 \mathrm{day}}\right)^{\delta} \\
\tau_{\text {distant }}=K_{4}\left(\frac{\nu}{5 \mathrm{GHz}}\right)^{-2.1}
\end{gathered}
$$

and

$$
\tau_{\mathrm{CSM}_{\mathrm{c} 1 \mathrm{umps}}}=K_{3}\left(\frac{\nu}{5 \mathrm{GHz}}\right)^{-2.1}\left(\frac{t-t_{0}}{1 \text { day }}\right)^{\delta^{\prime}}
$$

with $K_{1}, K_{2}, K_{3}$, and $K_{4}$ determined from fits to the data and corresponding, formally, to the flux density $\left(K_{1}\right)$, homogeneous $\left(K_{2}, K_{4}\right)$, and clumpy or filamentary $\left(K_{3}\right)$ absorption at $5 \mathrm{GHz}$ one day after the explosion date

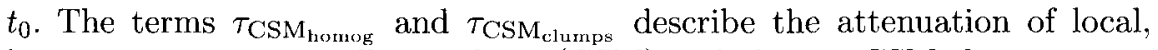
homogeneous circumstellar medium (CSM) and clumpy CSM that are near enough to the $\mathrm{SN}$ progenitor that they are altered by the rapidly expanding SN blastwave. The $\tau_{\mathrm{CSM}_{\mathrm{homog}}}$ absorption is produced by an ionized medium that completely covers the emitting source ("homogeneous external absorption"), and the $\left(1-e^{-\tau_{\mathrm{CSM}} \mathrm{clumps}}\right) \tau_{\mathrm{CSM}_{\mathrm{clumps}}}^{-1}$ term describes the attenuation produced by an inhomogeneous medium ("clumpy absorption"; see [8] for a 

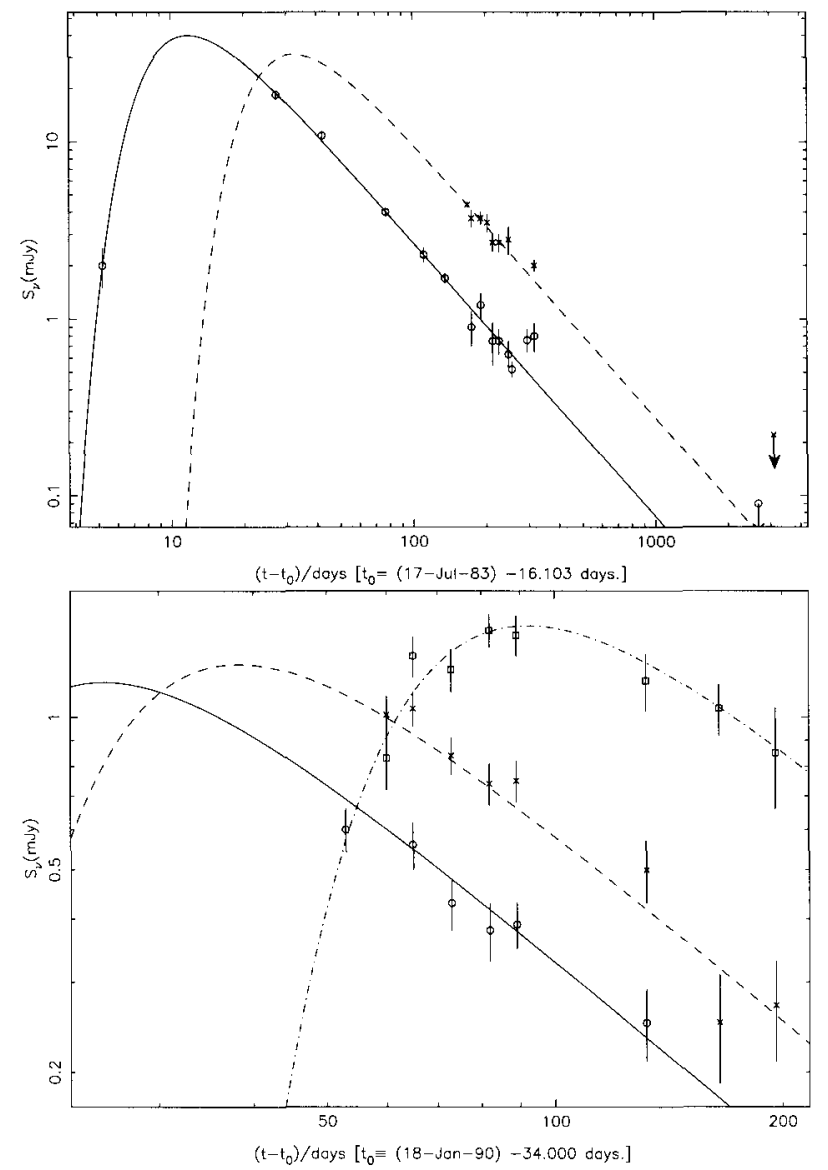

Fig. 2. Top (2a): type Ib SN1983N at $6 \mathrm{~cm}(4.9 \mathrm{GHz}$; open circles, solid line) and $20 \mathrm{~cm}(1.5 \mathrm{GHz} ;$ stars, dashed line). Bottom (2b): type Ic SN1990B. at $3.6 \mathrm{~cm}(8.4$ $\mathrm{GHz}$; open circles, solid line), $6 \mathrm{~cm}(4.9 \mathrm{GHz}$; stars, dashed line $)$, and $20 \mathrm{~cm}(1.5$ $\mathrm{GHz}$; open squares, dash-dot line).

more detailed discussion of attenuation in inhomogeneous media). The $\tau_{\text {distant }}$ term describes the attenuation produced by a homogeneous medium which completely covers the source but is so far from the SN progenitor that it is not affected by the expanding SN blastwave and is constant in time. All external and clumpy absorbing media are assumed to be purely thermal, singly ionized gas which absorbs via free-free (f-f) transitions with frequency dependence $\nu^{-2.1}$ in the radio. The parameters $\delta$ and $\delta^{\prime}$ describe the time dependence of the optical depths for the local homogeneous and clumpy or filamentary media, respectively. 
The f-f optical depth outside the emitting region is proportional to the integral of the square of the CSM density over the radius. Since in the simple Chevalier model the CSM density (constant mass-loss rate, constant wind velocity) decreases as $r^{-2}$, the external optical depth will be proportional to $r^{-3}$, and since the blastwave radius increases as a power of time, $r \propto t^{m}$ with $m \leq 1$ (i.e., $m=1$ for undecelerated blastwave expansion), it follows that the deceleration parameter, $m$, is

$$
m=-\delta / 3 .
$$

The model by Chevalier $[1,2]$ relates $\beta$ and $\delta$ to the energy spectrum of the relativistic particles $\gamma(\gamma=2 \alpha-1)$ by $\delta=\alpha-\beta-3$ so that, for cases where $K_{2}=0$ and $\delta$ is, therefore, indeterminate, one can use

$$
m=-(\alpha-\beta-3) / 3 .
$$

Since it is physically realistic and may be needed in some RSNe where radio observations have been obtained at early times and high frequencies, Eq. (1) also includes the possibility for an internal absorption term. This internal absorption $\left(\tau_{\text {internal }}\right)$ term may consist of two parts - synchrotron self-absorption (SSA; $\tau_{\text {internalssA }}$ ), and mixed, thermal f-f absorption/nonthermal emission $\left(\tau_{\text {internal }_{\mathrm{ff}}}\right)$.

$$
\begin{gathered}
\tau_{\text {internal }}=\tau_{\text {internal }_{\mathrm{SSA}}}+\tau_{\text {internal }_{\mathrm{ff}}} \\
\tau_{\text {internal }_{\mathrm{SSA}}}=K_{5}\left(\frac{\nu}{5 \mathrm{GHz}}\right)^{\alpha-2.5}\left(\frac{t-t_{0}}{1 \text { day }}\right)^{\delta^{\prime \prime}} \\
\tau_{\text {internal }_{\mathrm{ff}}}=K_{6}\left(\frac{\nu}{5 \mathrm{GHz}}\right)^{-2.1}\left(\frac{t-t_{0}}{1 \text { day }}\right)^{\delta^{\prime \prime \prime}}
\end{gathered}
$$

with $K_{5}$ corresponding, formally, to the internal, non-thermal $\left(\nu^{\alpha-2.5}\right)$ SSA and $K_{6}$ corresponding formally to the internal thermal $\left(\nu^{-2.1}\right)$ free-free absorption mixed with nonthermal emission, at $5 \mathrm{GHz}$ one day after the explosion date $t_{0}$. The parameters $\delta^{\prime \prime}$ and $\delta^{\prime \prime \prime}$ describe the time dependence of the optical depths for the SSA and f-f internal absorption components, respectively.

A cartoon of the expected structure of an $\mathrm{SN}$ and its surrounding media is presented in Fig. 1 (see also [4]). The radio emission is expected to arise near the blastwave [3].

\section{Radio Supernova Light Curves}

The success of the basic parameterization and modeling is shown in the good agreement between the model fits and the data for all subtypes of RSNe. The 

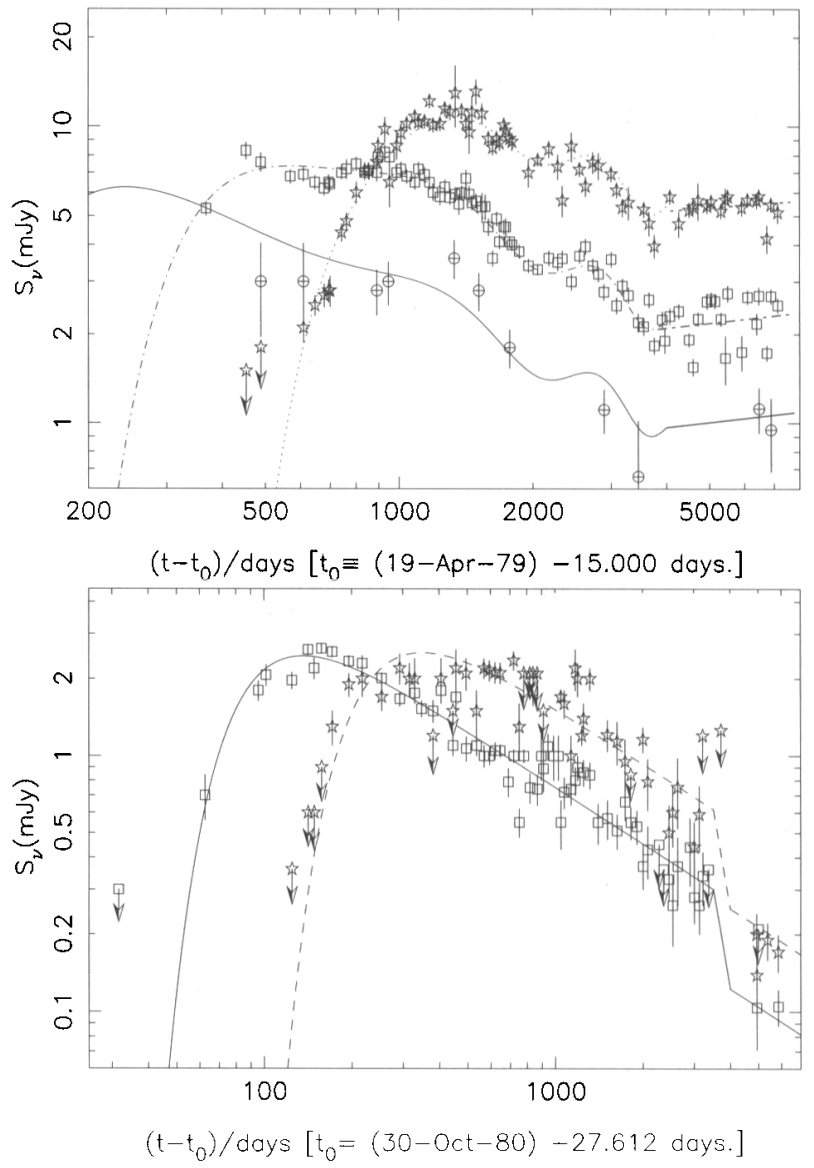

Fig. 3. Top (3a): type II SN1979C at $2 \mathrm{~cm}(14.9 \mathrm{GHz}$; crossed circles, solid line), 6 $\mathrm{cm}(4.9 \mathrm{GHz}$; open squares, dash-dot line $)$, and $20 \mathrm{~cm}(1.5 \mathrm{GHz}$; open stars, dotted line) Bottom (3b): type II SN1980K at $6 \mathrm{~cm}$ (4.9 GHz; open squares, solid line), and $20 \mathrm{~cm}(1.5 \mathrm{GHz}$; open stars, dashed line $)$.

observed data and the best fit model for the radio light curve of a Type Ib supernova, SN1983N [9] is shown in Fig. 2a; the radio light curve for a Type Ic supernova, SN1990B [11] is shown in Fig. 2b. For Type II supernovae, the data and model light curves are shown for SN1979C in Fig. 3a [7, 14, 15] and for SN1980K in Fig. 3b [6, 16]. Evidence for structure in the circumstellar medium can be seen in the optically thin, later portions of these latter two light curves. After day 4000, the radio emission from both SN1979C and SN1980K deviates from the simple model and SN1979C shows a sinusoidal modulation in its flux density prior to day $\sim 4000$. 
Thus, the radio emission from SNe appears to be relatively well understood in terms of blastwave interaction with a structured CSM as described by the Chevalier $[1,2]$ model and its extensions by $[5,12,13,18]$.

Acknowledgement. KWW wishes to thank the Office of Naval Research (ONR) for the 6.1 funding supporting this research. Additional information and data on radio supernovae can be found on $h t t p: / / r s d-w w w . n r l$ navy.mil/7213/weiler/ and linked pages.

\section{References}

1. R.A. Chevalier: Astrophys. J. 259, 302 (1982)

2. R.A. Chevalier: Astrophys. J. Lett. 259, L85 (1982)

3. R.A. Chevalier, C. Fransson: Astrophys. J. 420, 268 (1994)

4. T.A. Lozinskaya: In: Supernovae and Stellar Wind in the Interstellar Medium (American Institute of Physics, New York 1992) p. 190

5. M.J. Montes, K.W. Weiler, N. Panagia: Astrophys. J. 488, 792 (1997)

6. M.J. Montes, S.D. Van Dyk, K.W. Weiler, R.A. Sramek, N. Panagia: Astrophys. J. 506, 874 (1998)

7. M.J. Montes, K.W. Weiler, S.D. Van Dyk, R.A. Sramek, N. Panagia, R. Park: Astrophys. J. 532, 1124 (2000)

8. A. Natta N. Panagia: Astrophys. J. 287, 228 (1984)

9. R.A. Sramek, N. Panagia, K.W. Weiler: Astrophys. J. Lett. 285, L59 (1984)

10. R.A. Sramek, K.W. Weiler: Radio Supernovae. In: Supernovae and GammaRay Bursters, ed by K.W. Weiler (Springer-Verlag, Berlin Heidelberg New York 2003) pp. 145-169

11. S.D. Van Dyk, R.A. Sramek, K.W. Weiler, N. Panagia: Astrophys. J. 409, 162 (1993)

12. K. Weiler, R. Sramek, N. Panagia, J. van der Hulst, M. Salvati: Astrophys. J. 301, 790 (1986)

13. K.W. Weiler, N. Panagia, R.A. Sramek: Astrophys. J. 364, 611 (1990)

14. K. Weiler, S. Van Dyk, N. Panagia, R. Sramek, J. Discenna: Astrophys. J. 380, 161 (1991)

15. K. Weiler, S. Van Dyk, J. Pringle, N. Panagia: Astrophys. J. 399, 672 (1992)

16. K. Weiler, S. Van Dyk, N. Panagia, R. Sramek: Astrophys. J. 398, 248 (1992)

17. K.W. Weiler, N. Panagia, M.J. Montes: Astrophys. J. 562, 670 (2001)

18. K.W. Weiler, N. Panagia, M.J. Montes, R.A. Sramek: Ann. Rev. Astron. Astrophys. 40, 387 (2002) 